lassen sind. Denn dies würde eine dopaminerge Überstimulation bedeuten, die unweigerlich zur Augmentation führt, das heißt einer paradoxen Verschlechterung. Anders als beim Morbus Parkinson handelt es sich um Patienten mit intaktem dopaminergem System ohne Dopamindefizit. Informieren Sie auch den Patienten darüber, dass er keinesfalls eigenmächtig die Dosis steigern soll, wenn die Wirkung der Medikation nachlässt. Vielmehr soll er sich in diesem Fall bei seinem Arzt vorstellen, da die Therapie geändert werden muss.

Zu den drei für die RLS-Therapie zugelassenen Dopaminagonisten gehört auch transdermales Rotigotin (Neupro ${ }^{\oplus}$ ). Mit einer Dosis von $2 \mathrm{mg}$ Rotigotin wird in der
Regel der optimale Effekt erreicht. Zugelassen sind maximal $3 \mathrm{mg}$. Das Pflaster ermöglicht eine kontinuierliche dopaminerge Stimulation, die mit einem vergleichsweise geringen Augmentationsrisiko assoziiert ist. Rotigotin weist als einzige dopaminerge Therapie auch Langzeitdaten auf. Bei Patienten mit mittelschwerem bis schwerem idiopathischen RLS hat Rotigotin eine konstante Wirksamkeit über fünf Jahre gezeigt.

Angelika Bischoff

Symposium „Epilepsie und Bewegungsstörungen - Therapie und Patientenbedürfnisse", 88. DGN-Kongress, Düsseldorf, 24.9.2015; Veranstalter: UCB Pharma

\title{
Depression, beeinträchtigtes Funktionsniveau, Anhedonie - was zählt für den Patienten?
}

- Patientenrelevante Parameter wie die Linderung der Anhedonie stellen heute neben der Verbesserung der depressiven Kernsymptome und des psychosozialen Funktionsniveaus eines der wichtigsten Ziele der antidepressiven Therapie dar.

Wenn es darum gehe, Patienten mit Depression in Remission zu bringen, scheine Agomelatin $\left(\right.$ Valdoxan ${ }^{\circledast}$ ) die effektivste Therapieoption zu sein, berichtete Professor David Taylor, Leiter des Pharmacy Departments am Maudsley Hospital in London. Darauf deuten die Ergebnisse einer aktuellen Netzwerk-Metaanalyse hin [Khoo AL et al. CNS Drugs 2015 Aug 21. Epub ahead of print]. In die Analyse flossen die Daten von 76 klinischen Studien zur Wirksamkeit und Verträglichkeit von insgesamt zehn verschiedenen Antidepressiva ein. In Bezug auf die Remissionsrate erwies sich Agomelatin von allen untersuchten Antidepressiva als die Substanz mit der höchsten Wirksamkeit und besten Verträglichkeit.

Mittlerweile hat sich Agomelatin auch unter den Bedingungen des klinischen Alltags bewährt und sich auch bei Patienten mit Komorbiditäten als effektiv und verträglich erwiesen, berichtete Professor Kai Kahl, leitender Oberarzt der Klinik für Psychiatrie an der Medizinischen Hochschule Hannover $(\mathrm{MHH})$. Erste, noch nicht veröffentlichte Ergebnisse einer derzeit an der $\mathrm{MHH}$ laufenden Untersuchung, in deren Rahmen 30
Patienten mit Depression und kardialen/ neurologischen Komorbiditäten Agomelatin erhielten, seien positiv. Sechs Wochen nach Therapiebeginn betrug die Responderrate $53,3 \%$ und die Remissionsrate 43,3\%. Körpergewicht, EKG und Blutzuckerwerte seien stabil geblieben, und es sei keine Dosisanpassung der Grundmedikation erforderlich gewesen, so Kahl.

Die Ergebnisse einer nichtinterventionellen Studie bei 1.513 Patienten zeigen, dass Agomelatin auch die depressionsbedingte Anhedonie (erfasst mit der Snaith Hamilton Pleasure Scale; SHAPS) und das psychosoziale Funktionsniveau (erfasst mit der Sheehan-Disability-Scale (SDS) verbessert. Dabei ergab sich eine starke Korrelation zwischen den SHAPS- und SDS-Scores $(r=0,642$; $p<0,0001$ ) [Llorca PR, Gourion D. Eur Neuropsychopharmacol 2014; 24 (Suppl): S468]. Die Effekte auf die Anhedonie und das Funktionsniveau sind laut Kahl auf die synergistische Wirkung der Substanz als melatonerger $\mathrm{MT}_{1} / \mathrm{MT}_{2}$-Agonist und serotonerger $5-\mathrm{HT}_{2 c}$-Antagonist zurückzuführen.

Abdol A. Ameri

Satellitensymposium "Interest in and enthusiasm for meta-analyses", 28. ECNP-Kongress, Amsterdam, 30.8.2015;

Veranstalter: Servier Deutschland

\section{Zulassungsempfehlung}

Das erste und einzige spezifische Antidot (Idarucizumab) für ein direktes orales Antikoagulans hat eine positive Zulassungsempfehlung erhalten. Der Ausschuss für Humanarzneimittel der EMA - CHMP - stützt sich auf die Ergebnisse von klinischen Daten, die die Aufhebung der Dabigatran (Pradaxa ${ }^{\oplus}$ )-induzierten Gerinnungshemmung innerhalb weniger Minuten zeigen.

Nach Informationen von Boehringer Ingelheim

\section{Zulassungserweiterung I}

Die Depot-Formulierung von Aripiprazol (Abilify Maintena ${ }^{\oplus}$ ) hat eine Zulassungserweiterung erhalten. Das Präparat kann jetzt als monatliche Injektion sowohl in den Gluteal- als auch in den Deltamuskel angewendet werden.

Nach Informationen von Lundbeck/Otsuka

\section{Zulassungserweiterung II}

Das $8 \%$ ige Capsaicin-Pflaster Qutenza ${ }^{\mathrm{TM}}$ wurde 2009 in der EU zur Behandlung peripherer neuropathischer Schmerzen bei nichtdiabetischen Erwachsenen als Monotherapie oder in Kombination mit anderen Schmerzmitteln zugelassen. Nun hat die Europäische Arzneimittelagentur (EMA) die Zulassung von Qutenza ${ }^{\mathrm{TM}}$ auf die Behandlung erwachsener Patienten mit Diabetes erweitert.

Nach Informationen von Astellas

\section{Preiswürdige Spitzenforschung}

Die Gewinner des von Novartis mit insgesamt $100.000 €$ dotierten OppenheimFörderpreises für Multiple Sklerose wurden anlässlich des 88. DGN-Kongresses gekürt. Dr. Helena Radbruch, Berlin, erhielt den Preis in der Kategorie "Klinik" für ihr anspruchsvolles Projekt zur Charakterisierung von inflammatorischen JCV-Infektionen des ZNS. In der Kategorie „Präklinik“ überzeugte Dr. Klaus Lehmann-Horn, München, die Jury mit einem hochaktuellen Ansatz zur Untersuchung der Rolle von B-Zell-Agglomerationen bei sekundär progredienter MS.

Nach Informationen von Novartis 